\title{
Die Entwicklung der Homöopathie in den Vereinigten Staaten*
}

Josef M.Schmidt

\section{SummaRY}

After an enormous spread in the United States of America during the 19th century homeopathy had almost completely vanished from the scene by the beginning of the 20th century. For the past two decades, however, it seems once again to experience a kind of renaissance. Major aspects of this development-in terms of medical and cultural history, sociology, politics, and economics-are illustrated on the basis of a general history of homeopathy in the United States. Using original sources, a first attempt is made to reconstruct the history of homeopathy in San Francisco which has some institutional peculiarities that make it unique within the whole country.

\section{Zusammenfassung}

Auf die rasche Verbreitung der Homöopathie in den USA im 19. Jahrhundert folgte ihr fast vollständiger Niedergang zu Beginn des 20. Jahrhunderts, an den sich seit etwa zwei Jahrzehnten eine Art Renaissance dieser Heilmethode anzuschliessen scheint. Die wichtigsten medizin- und kulturhistorischen, soziologischen, politischen und ökonomischen Faktoren dieser Entwicklung werden zunächst anhand der allgemeinen Geschichte der Homöopathie in den USA darge-

\footnotetext{
* Im Rahmen eines Forschungsstipendiums der Deutschen Forschungsgemeinschaft hatte der Autor 1991/92 die Möglichkeit, den Ursachen und Hintergründen des Aufstiegs, Niedergangs und Fortbestehens der Homöopathie in den USA bzw. speziell in San Francisco nachzugehen, wo noch Anfang dieses Jahrhunderts ein homöopathischer Lehrstuhl an einer Staatsuniversität eingerichtet worden war, der bis in die 50er Jahre hinein bestand. - Obwohl die Auswertung des Materials (Archivalien, Interviews usw.) noch nicht abgeschlossen ist, können hier bereits erste Erkenntnisse aus dieser Studie einem grösseren Publikum zugänglich gemacht werden.
} 
stellt. Anschliessend wird erstmals versucht, anhand von Originalquellen die besondere Geschichte der Homöopathie in San Francisco zu rekonstruieren, die aufgrund ihrer vor allem institutionellen Besonderheiten einzigartig innerhalb des ganzen Landes ist.

Sowohl ihren steilsten Aufstieg als auch ihren tiefsten Fall hat die Homöopathie in den Vereinigten Staaten von Amerika erlebt: nachdem dort zur Zeit ihrer weitesten Verbreitung (vor etwa 100 Jahren) jeder achte Arzt von einem homöopathischen College graduiert worden war $^{1}$, hatte die Homöopathie gegen Mitte dieses Jahrhunderts praktisch keine nennenswerte Bedeutung mehr im amerikanischen Gesundheitswesen ${ }^{2}$. Erst in jüngster Zeit ist sie, als eine der neuerdings von weiten Bevölkerungskreisen favorisierten «alternativen» Heilmethoden, wieder zu einem politisch und ökonomisch ernstzunehmenden Faktor geworden. Ihre Popularität ist gross, und der Jahresumsatz der homöopathischen pharmazeutischen Industrie liegt derzeit bei $\$ 100$ Millionen, was immerhin etwa einem Promille des Umsatzes der «regulären» pharmazeutischen Industrie (etwa \$60 Milliarden) entspricht ${ }^{3}$.

Im folgenden soll nun versucht werden, einen historischen Überblick über die wichtigsten Etappen und Determinanten dieser Entwicklung zu geben.

\section{Die Geschichte der Homöopathie in den USA}

Die Homöopathie, begründet von Samuel Hahnemann (1755-1843) in den Jahren von 1796 bis $1810^{4}$, kam in den 20er Jahren des 19. Jahrhunderts auf zwei verschiedenen Wegen nach Amerika: erstens über den amerikanischen Arzt dänischer Abstammung Hans Burch Gram (1786-1840) 1825 nach New York und zweitens über den deutschen Arzt Wilhelm Wesselhoeft (17941858) und den Schweizer Arzt Heinrich Detweiler (1795-1887) 1828 nach Pennsylvania, wo - unter Mitwirkung des 1833 immigrierten deutschen Arztes Constantin Hering (1800-1880) - in Allentown 1835 die deutschsprachige «Nordamerikanische Akademie der homöopathischen Heilkunst» gegründet wurde ${ }^{5}$.

In diesen ersten Jahrzehnten des 19. Jahrhunderts waren in Amerika in der Medizin einerseits - noch extremer als in Europa - die heroischen Praktiken des Aderlassens, Purgierens usw. (Benjamin Rush [1745-1813] 
u. a.) vorherrschend, andererseits gab es als Alternativen hierzu die «Indian Doctors», die «Botanics» sowie den Thomsonianismus (Samuel Thomson [1769-1843]). Die beiden letzteren Richtungen verschmolzen in den 40er Jahren zur sogenannten Eklektischen Schule. Obwohl sich die homöopathischen Ärzte von den Anhängern dieser Sekten grundlegend dadurch unterschieden, dass sie über dieselbe medizinische Schul-Ausbildung verfügten wie ihre «regulären» Kollegen, wurden sie von diesen von Anfang an als zu diesen Gruppen von Aussenseitern gehörig betrachtet ${ }^{6}$.

Nachdem 1844 als erste landesweite ärztliche Organisation das «American Institute of Homeopathy» gegründet worden war, organisierten sich 1847 auch die regulären Ärzte in der «American Medical Association» (AMA) und verboten gleichzeitig in ihrem «Code of Ethics» jegliche Zusammenarbeit mit homöopathischen Ärzten, denen von da an reguläre Institutionen verschlossen waren ${ }^{7}$. Anstatt, wie erwartet, als eigene medizinische Schule auszusterben, gelang es den Homöopathen und ihren Anhängern nun allerdings, begünstigt durch die liberale Gesetzgebung, eigene Colleges, Krankenhäuser, Apotheken usw. zu gründen, die bis zum Ende des 19. Jahrhunderts eine ansehnliche Zahl erreichten: 1898 waren es 20 homöopathische Colleges, 66 allgemeine und 74 spezialisierte homöopathische Krankenhäuser, 57 homöopathische Apotheken, 9 nationale homöopathische Gesellschaften, 33 Staats-, 85 lokale und 39 andere homöopathische Gesellschaften $^{8}$. Das erste wie auch das letzte der zum Teil recht kurzlebigen insgesamt über 100 verschiedenen homöopathischen Colleges war das 1848 inkorporierte «Homeopathic Medical College of Pennsylvania», aus dem inzwischen das renommierte Hahnemann University Medical Center hervorgegangen ist ${ }^{9}$.

Die beträchtliche Popularität und finanzielle Unterstützung der sich damals rasch ausbreitenden Homöopathie erklären manche Autoren damit, dass jene - «sophisticated» und europäischer Herkunft - im Gegensatz zu den anderen Sekten jener Zeit vor allem die vermögenden und einflussreichen Kreise der Mittel- und Oberschicht der grossen Städte ansprach ${ }^{10}$. Ein weiterer wesentlicher Faktor war zweifellos aber auch die philosophische Nähe zwischen Homöopathie und dem damals in Amerika relativ weit verbreiteten Swedenborgianismus, was sich konkret so äusserte, dass sowohl zahlreiche bedeutende Homöopathen bekannte Swedenborgianer (Hering, Kent, Boericke u.a.) waren als auch umgekehrt Geistliche und sonstige Anhänger dieser Sekte, also medizinische Laien, als eifrige Verbreiter der Homöopathie fungierten ${ }^{11}$. Von einzelnen Historikern wird behauptet, dass 
sich die Anhängerschaft der Homöopathie zu grossen Teilen aus früheren Thomsonianisten rekrutierte, doch sprechen etwa ebenso viele Argumente auch gegen diese These ${ }^{12}$. Medizinisch-psychologische Gründe für die grosse Akzeptanz der Homöopathie von seiten der Bevölkerung bestanden wiederum in der guten Verträglichkeit der homöopathischen Mittel, den relativ geringen Nebenwirkungen und der durch die methodische Vorschrift des Individualisierens vorgegebenen grösseren Zuwendung des Arztes zu seinen Patienten.

In der zweiten Hälfte des 19. Jahrhunderts näherten sich die beiden getrennt voneinander existierenden und sich bekämpfenden medizinischen Schulen zunehmend einander an: Die reguläre Medizin verliess die heroischen Praktiken vergangener Jahrzehnte, verwandte vermehrt Einzelmittel sowie geringere Dosen von Medikamenten und übernahm zahlreiche Substanzen aus der homöopathischen Materia medica in ihren eigenen Arzneischatz ${ }^{13}$. Andererseits benutzten immer mehr Homöopathen auf eine empirische und eklektische Weise oft dieselben regulären Fertigpräparate unbekannten Inhalts wie ihre «allopathischen» Kollegen. Die Folgen dieser Annäherung waren einerseits 1881 die Gründung der «International Hahnemannian Association», mit der sich die Puristen unter den Homöopathen von ihren «eklektischen» Kollegen distanzierten. Andererseits führte die Tendenz von seiten zahlreicher regulärer Ärzte, das nach wie vor bestehende Verbot der Zusammenarbeit mit homöopathischen Kollegen zunehmend laxer auszulegen, 1882 zu einer Spaltung der sich damals unter der AMA erst langsam konstituierenden regulären ärztlichen Profession - die erst 1903 wieder vereint werden konnte, nachdem der alte «Code of Ethics» von 1847 völlig revidiert worden war $^{14}$.

Als Hauptursache für den letztendlichen Untergang der homöopathischen Schulen werden als erstes meist die grossen Entdeckungen und Fortschritte der naturwissenschaftlichen Medizin, vor allem der Pathologie und Bakteriologie, gegen Ende des Jahrhunderts genannt. Obwohl die diesbezüglichen Forschungsergebnisse zunächst noch kaum Konsequenzen für die Therapie hatten, waren diese Errungenschaften langfristig zweifellos ein wesentlicher Faktor der weiteren Entwicklung. Dennoch erscheint es durchaus vorstellbar, dass die Geschichte unter anderen soziologischen, ökonomischen und politischen Randbedingungen anders hätte verlaufen können.

Zieht man etwa die Geschichte des medizinischen Ausbildungswesens mit heran, so zeigt sich zum Beispiel, dass die medizinischen Schulen, die von 
den zu Beginn des 19. Jahrhunderts noch allein für die Lizenzierung von Ärzten zuständigen medizinischen Gesellschaften geschaffen worden waren, sich gegen Mitte des Jahrhunderts rasch verselbständigten und zunehmend in Konflikt mit letzteren gerieten. Auf der Seite der zahlreichen privaten, von Studiengebühren abhängigen und miteinander um die Gunst der Studenten konkurrierenden Schulen bestand primär ein Interesse an möglichst grossen Studentenzahlen sowie an möglichst einzigartigen Professoren. Dem stand das Interesse der Gesellschaften an einer Beschränkung der Ärztezahl und an einer Standardisierung der ärztlichen Ausbildung gegenüber. In diesem Konfliktfeld vollzog sich die Formierung der AMA sowie die Professionalisierung der amerikanischen Ärzteschaft. Gegen Ende des Jahrhunderts kristallisierten sich schliesslich als gemeinsame Ziele sowohl der medizinischen Gesellschaften und Lizenzierungs-Gremien als auch eines führenden Blocks von Colleges folgende heraus: Anhebung der Aufnahmebedingungen, Spezifizierung der Curricula, Beseitigung der finanzschwächsten privaten Schulen und Reduzierung der Zahl der Absolventen ${ }^{15}$.

Begünstigt wurde die nun folgende landesweite medizinische Ausbildungs-Reformbewegung durch die Expansion der Städte, den Ausbau an Kommunikationsmitteln, den Eindruck der wissenschaftlichen Erfolge der Medizin in Deutschland und Frankreich, wo es wesentlich weniger medizinische Fakultäten, dabei aber wesentlich mehr Vollzeit-Professoren gab, sowie den nationalen Wunsch nach Unabhängigkeit von Europa auch in der medizinischen Wissenschaft. Finanziell ermöglicht wurde die Reform aber durch die etwa um die Jahrhundertwende entstandenen grossen Stiftungen amerikanischer Philanthropen, deren bedeutendste die Carnegie Foundation (1906) und die Rockefeller Foundation (1913) waren. So besassen zum Beispiel alle medizinischen Colleges zusammen 1891 ein Vermögen von knapp \$ 1/2 Mill.; demgegenüber gaben zwischen 1903 und 1934 neun Stiftungen allein $\$ 150$ Mill. an medizinische Institutionen ${ }^{16}$.

Zunächst wurde 1893 Johns Hopkins Medical School nach dem Vorbild deutscher Universitäten mit Vollzeit-Fakultät, modernen Labors, Forschungseinrichtungen und anderem ausgestattet ${ }^{17}$. Ab 1896 wurden dann im Journal der AMA jährlich ein Bericht über sämtliche medizinischen Colleges der USA publiziert, wo die Aufnahmebedingungen, Studentenzahlen, Zahl der im Lizenzierungsverfahren durchgefallenen Kandidaten und ähnliches öffentlich gemacht wurden. Dieses 1883 gegründete Journal erwies sich als eines der bedeutendsten Werkzeuge, mit denen die AMA gegen 
Anfang des 20. Jahrhunderts letztlich zum eindeutigen Vertreter der nun erst geeinten und zentralisierten ärztlichen Profession wurde ${ }^{18}$. Die Mitgliederzahl der medizinischen Gesellschaften, die an die AMA angeschlossen waren, verdoppelte sich von 1901 bis 1908 von 35000 auf $70000^{19}$.

Für dieselbe Zeit lässt sich auch die an Bedeutung zunehmende ökonomische Rolle der aufblühenden pharmazeutischen Industrie für die Entwicklung der AMA aufzeigen: Während laut Code of Ethics von 1847 sowie einem Beschluss der Treuhänder des Journal der AMA von 1883 Anzeigen von Fertigpräparaten zunächst verboten waren, wurde dies - nachdem Ideal und Wirklichkeit bereits weit auseinanderklafften - 1895 formal revidiert, woraufhin sich in den nächsten zehn Jahren das Einkommen aus Anzeigen verzehnfachte und 1909 mit $\$ 150000$ pro Jahr bereits die Haupteinnahmequelle der AMA geworden war ${ }^{20}$.

1906-1907 liess das 1905 gegründete «AMA Council on Medical Education» die einzelnen Colleges von Vertretern der AMA sowie der Lizenzierungs-Behörden besuchen und in Qualitäts-Klassen einteilen. Dieses Unternehmen wurde schliesslich vereint mit der 1906 gegründeten «Carnegie Foundation for the Advancement of Teaching» und das Ergebnis 1910 durch Abraham Flexner veröffentlicht ${ }^{21}$ - was weitreichende Folgen hatte.

Gemessen am neu festgelegten Standard, der sich an Johns Hopkins Medical School als Modell orientierte, hatte die Mehrzahl der damals existierenden Colleges nun keine Existenzberechtigung mehr. 20 Schulen schlossen angeblich noch vor der Publikation des Reports, um in diesem gar nicht erst erwähnt zu werden, und 92 weitere fusionierten entweder mit anderen oder schlossen zwischen 1904 und 1915 angesichts der Diskrepanz zwischen den nun festgesetzten Standards und ihren finanziellen Möglichkeiten für immer ihre Pforten - darunter relativ viele homöopathische Colleges sowie fast alle «schwarzen» Colleges. Da man keine mögliche Rolle für die kleineren privaten Schulen sah, wurden praktisch alle finanziellen Mittel auf den Ausbau vor allem der grossen, renommierten Universitäten konzentriert. Während der wirtschaftlichen Depression verringerten sich die Zuschüsse aus Stiftungsgeldern zwar wieder, doch war bis dahin bereits die moderne Struktur von grossen, gutausgerüsteten medizinischen Zentren unwiderruflich etabliert ${ }^{22}$.

Obwohl John D. Rockefeller senior sich selbst homöopathisch behandeln liess und in Briefen ausdrücklich wünschte, dass es weiterhin auch homöopathische Schulen geben solle, kanalisierten sein Sohn sowie dessen Berater 
Starr J. Murphy und Frederick T. Gates die Millionen der Stiftung so, dass keines der homöopathischen Colleges etwas davon erhielt. Bis 1960 sind insgesamt ca. \$600 Mill. in die Reform der regulären medizinischen Colleges geflossen; demgegenüber verfügten von den letzten homöopathischen Colleges etwa das Hahnemann Medical College of Philadelphia 1921 über ein Gesamtvermögen von $\$ 325000$ und das Hahnemann College of Chicago über $\$ 537000^{23}$.

Kritiker der damaligen politischen Entscheidungen sprechen heute von «antihomeopathic bias» des Flexner-Reports, mit dem Argument, dass sowohl die Kriterien des damals festgesetzten medizinischen Standards speziell die homöopathischen Colleges benachteiligten, als auch Flexner selbst in seinem Report, der dann die Grundlage für die Entscheidungen von seiten der Stiftungen über die Förderungswürdigkeit einzelner Projekte bildete, offen gegen die Homöopathie Partei ergriff ${ }^{24}$. Von den 22 homöopathischen Colleges von 1900 gab es 1913 noch zehn, 1919 noch fünf, 1923 noch zwei - die schliesslich innerhalb der nächsten Jahrzehnte den Unterricht in Homöopathie endgültig einstellten ${ }^{25}$. Das letzte homöopathische Diplom wurde 1950 in Philadelphia ausgestellt ${ }^{26}$.

Als absehbar wurde, dass es innerhalb der Universitäten für die Homöopathen keinen Nachwuchs mehr geben konnte, gründeten sie 1921 die «American Foundation for Homeopathy», deren Aufgabe es sein sollte, eine Postgraduierten-Weiterbildung für Ärzte zu organisieren. Die fortan jährlich durchgeführten Sommerkurse in Millersville, Pennsylvania, erwiesen sich jedoch als weitaus ineffektiver als die bisherige studienbegleitende homöopathische Ausbildung von Studenten ${ }^{27}$. 1974 ging aus dieser Foundation das «National Center for Homeopathy» hervor, das nun auch Laien einbezog und dessen Konzept der «Affiliated Study Groups» sich so grosser Beliebtheit erfreute, dass es heute davon bereits über 155 (Minimum 10 Personen) in den USA gibt und die Gesamtmitgliederzahl der Organisation bei über 6000 liegt. Im Gegensatz zur neuerlichen Verbreitung der Homöopathie unter Laienpraktikern liegt die Zahl der organisierten homöopathischen Ärzte allerdings lediglich bei etwas über 100 für das ganze Land ${ }^{28}$.

Homöopathische Arzneimittel können seit dem Food, Drug and Cosmetic Act von 1938 - bis auf einzelne Ausnahmen - ohne Rezept in Drogerien gekauft werden (OTC-drugs, = over the counter), sind in ihrem Status als Medikamente von den modernen Standards eines möglicherweise verlangten Wirksamkeitsnachweises heute aber zunehmend bedroht. 


\section{Die Geschichte der Homöopathie in San Francisco}

Innerhalb dieses nationalen Rahmens spielte sich auch die lokale Geschichte der Homöopathie in San Francisco ab. Naturgemäss beginnt diese Geschichte erst während der Zeit des Goldrauschs, als mit der ersten grossen Einwanderungswelle der «1849er» allerdings bereits etwa 1300 bis 1500 Ärzte unterschiedlichster Herkunft und Überzeugung nach Kalifornien kamen, was bald nicht nur zu einem starken Methodenpluralismus, sondern angeblich vorübergehend auch zu der höchsten Ärztedichte pro Patient der damaligen Welt führte ${ }^{29}$. Demgegenüber nahm sich die Zahl der ersten Homöopathen in Kalifornien eher gering aus. 1849 kam Benjamin Ober, 1850 Moritz Richter, der erste Homöopath San Franciscos, und 1853 praktizierten dort auch Eckel, Cushing, Bryant, Springsted und Kafka, von denen die meisten deutscher Herkunft waren. Diese ersten Homöopathen waren den übrigen kalifornischen Ärzten rechtlich zunächst gleichgestellt, bis in den 70er Jahren das Gerücht von einem geplanten, die medizinische Praxis reglementierenden Gesetz jene veranlasste, sich zu organisieren. So wurde 1871 die «California State Medical Society of Homeopathic Practitioners» gegründet, von der sich 1874 die «Pacific Homeopathic Medical Society of the State of California» abspaltete. Um die Differenzen zwischen der älteren mehr eklektischen und der neueren strikteren Gesellschaft zu versöhnen, wurde 1877 die «California State Homeopathic Medical Society» gegründet, der 1878 schliesslich als einziger von den dreien das Recht der Lizenzierung homöopathischer Ärzte zugesprochen wurde und die bis heute besteht. 1885 waren von etwa 200 kalifornischen Homöopathen 56 ihre Mitglieder ${ }^{30}$.

Angesichts der geographischen Isolation und Entfernung von den übrigen homöopathischen Zentren des Landes wurde ab 1881 die Einrichtung eines eigenen homöopathischen Colleges geplant, 1882 mit dem «California Homoeopath» eine eigene Zeitschrift gegründet und schliesslich 1884 das «Hahnemann Medical College of San Francisco» eröffnet. Um auch ein eigenes Krankenhaus betreiben zu können, wurde die Bezeichnung des College 1887 in «Hahnemann Hospital College of San Francisco» abgewandelt. Ein im selben Jahr eröffnetes Krankenhaus wurde jedoch bereits ein Jahr danach wieder geschlossen - unter anderem aufgrund interner Streitigkeiten, mangelnder Unterstützung von seiten der homöopathischen Ärzte, der unzureichenden Bezahlung der Professoren, von denen die meisten ehrenamtlich unterrichteten, sowie «Schwierigkeiten mit der Nachbarschaft», die sich offenbar belästigt fühlte ${ }^{31}$. 
Nachdem das College weder einen festen Sitz noch die finanziellen Mittel hatte, ein eigenes Gebäude zu erwerben, wurde bereits 1892 zum ersten Mal ein Plan zur Affilierung mit der Universität vorgeschlagen, jedoch nicht weiter verfolgt. Trotz zu befürchtender finanzieller Einbussen entschloss sich das «Hahnemann Hospital College of San Francisco» bereits 1894 noch vor den meisten Colleges im Osten der USA - zu einem erweiterten Curriculum von fortan vier Jahren mit je sieben Monaten Dauer. Nach einer Zeit finanzieller Bedrängnis schlug man 1897 erneut einen Plan der Affilierung mit der Universität vor, der von dieser jedoch «aus ökonomischen Gründen» abgelehnt wurde. Durch diese Entscheidung eher stimuliert als entmutigt, konnten von den Homöopathen San Franciscos nun relativ rasch \$10000 für den Bau eines eigenen College-Gebäudes beschafft, 1899 der Grundstein gelegt und alsbald der neue Bau in Betrieb genommen werden ${ }^{32}$.

Ab 1896 wurde von dem homöopathischen Ärztepaar James W. und Florence Ward das Homeopathic Sanatorium in San Francisco betrieben, das zunächst 15 Betten hatte, sich 1899 aber vergrösserte und dessen erwirtschafteter Gewinn zum Teil dem späteren Hahnemann Hospital gestiftet wurde. Im Zuge der Planung des letzteren formierte sich das College 1902 unter einer neuen Satzung, die auch die Integrierung einer Ausbildungsschule für Krankenschwestern enthielt, zum nunmehrigen «Hahnemann Medical College of the Pacific» ${ }^{33}$. 1903 hatte man bereits $\$ 80000$ gesammelt, 1905 wurde der Grundstein gelegt und 1906 das Gebäude offiziell eröffnet. Das schon eine Woche später in San Francisco ausgebrochene Erdbeben und Feuer richtete jedoch auch hier beträchtlichen Schaden an, so dass die eigentliche Inbetriebnahme schliesslich erst 1907 erfolgen konnte. Das Krankenhaus hatte zunächst 130 und ab 1908140 Betten und wurde als mit den neuesten Ausrüstungen eingerichtet sowie als das modernste «up-todate Hospital on the Pacific Coast» beschrieben ${ }^{34}$.

Im Zusammenhang mit dem bereits erwähnten Flexner-Report, bei dem das Hahnemann Medical College of the Pacific nicht besonders gut abschnitt ${ }^{35}$, trennten sich 1910 das College und das Krankenhaus institutionell voneinander, indem letzteres fortan von der neu gegründeten «Hahnemann Hospital Corporation» verwaltet wurde. Ab 1915 begann das College, mit der University of California, Medical School, bezüglich einer Verschmelzung beider Schulen zu verhandeln. 1916 willigten beide Parteien in dieses «grosse Abenteuer Kaliforniens» ein, woraufhin das Eigentum des College an die Universität überging und zwei Professuren, eine für Homeopathic Materia Medica und eine für Applied Homeopathic Therapeutics an der Universität 
eingerichtet wurden ${ }^{36}$. Erstere Stelle vertrat ab 1915 William Boericke aus San Francisco ${ }^{37}$, letztere ab 1917 Anson Hill aus New York ${ }^{38}$. Nach drastischen Kürzungen von deren Gehältern durch die Universität 1920/21 (von $\$ 3000$ auf $\$ 600$ bzw. von $\$ 3200$ auf $\$ 1200$ pro Jahr $)^{39}$ traten diese zurück. Nachfolger von ersterem wurde 1921/22 sein Sohn Garth W. Boericke aus Philadelphia ${ }^{40}$, von letzterem Thomas McGavack und Paul Wyne.

Ein weiteres Verschmelzungs-Abkommen wurde 1918 zwischen der Universität und dem Hahnemann Hospital geschlossen, wonach dieses 1919 in den Besitz der Universität überging und von jener geführt und verwaltet wurde, wobei den Homöopathen allerdings das Recht, ihre Patienten dort selbst zu betreuen, erhalten blieb ${ }^{41}$. 1930 gab die Universität im Zusammenhang neuer feuerpolizeilicher Vorschriften, deren Erfüllung erhebliche Investitionen verlangt hätte, das Krankenhaus wieder an die Hospital Corporation der Homöopathen zurück, die das Gebäude 1931 abreissen und an derselben Stelle ein neues errichten liessen, das 1941 eröffnet wurde. 1971 wurde an dieser Stelle ein drittes Mal ein neuer Bau erstellt, 1975 der Name des Krankenhauses von «Hahnemann-Hospital» in «Marshal Hale Memorial Hospital» umgeändert ${ }^{42}$, und 1988 fusionierte dieses mit dem anliegenden Children's Hospital, das seinerseits 1991 mit dem Presbyterian Hospital zum heutigen California Pacific Medical Center verschmolz. Schon seit mehreren Jahrzehnten wird in dem Haus keine Homöopathie mehr betrieben.

Indirekt betroffen war hiervon auch die Entwicklung der HomöopathieLehrstühle an der Universität. Wie bereits 1920/21, wurden die Gehälter der beiden homöopathischen Professoren 1933 noch einmal drastisch gekürzt (von \$1500 und \$1200 auf insgesamt \$900 pro Jahr), mit der Begründung, dass die Zins-Erträge aus dem 1916 vom Hahnemann College übernommenen Homeopathic Teaching Fund nur noch $\$ 1000$ pro Jahr betrugen. Ausserdem würden die beiden Dozenten weder ihre volle Zeit ihrer Arbeit an der Universität widmen (jeder hatte eine eigene Praxis in der Stadt) noch hätten diese die akademischen Qualifikationen, die eine reguläre Professur an der Universität rechtfertigen würden ${ }^{43}$. McGavack trat daraufhin 1934 zurück ${ }^{44}$ unter dem Hinweis, bei dem geringen Gehalt finanziell vor allem auf seine Praxis angewiesen zu sein, sowie der Beschuldigung, dass man ihn an der Universität systematisch an seinen Forschungen zur Homöopathie gehindert habe ${ }^{45}$. Obwohl von seiten der Universitätsleitung durchaus «sympathetic interest in homeopathy» (Präsident Sproul) ${ }^{46}$ bzw. Wohlwollen einzelnen Homöopathen gegenüber (Dekan Porter) vorhanden war (einige liessen 
sich selbst bzw. ihre Familien sogar homöopathisch behandeln) ${ }^{47}$ und es -im Hinblick auf künftige Stifter - als nicht opportun erschien, das Abkommen von 1916 unter Rückgabe des Vermögens schon wieder zu lösen, stand 1935 in den erneuten Verhandlungen die diesbezügliche Entscheidung der Universität in Frage ${ }^{48}$. Wäre in der Zwischenzeit nicht Otto E.Guttentag aus Deutschland nach San Francisco gekommen, der eben jene akademischen Qualifikationen mitbrachte, die sich die Universität wünschte, hätte das 1936 schliesslich beschlossene revidierte Abkommen zwischen der Universität und dem Hahnemann Medical College of the Pacific, das nun genau auf die Person Guttentags zugeschnitten war, wohl nicht mehr zustande kommen können ${ }^{49}$. So wurde Otto E. Guttentag 1936 (full-time) Assistant Professor and Chair of Homeopathy und 1940 Associate Professor and Chair of Homeopathy ${ }^{50}$.

Da zum einen die eigentlich homöopathische Ausbildung Guttentags nicht sehr umfassend war, zum anderen bei den Studenten die Grundlagen für ein wirkliches Verständnis der homöopathischen Prinzipien angeblich nicht vorausgesetzt werden konnten, verlagerte Guttentag den Schwerpunkt seiner Vorlesungen von der konkreten homöopathischen Materia medica und Therapie bald auf die Behandlung allgemein anthropologischer, philosophischer und ethischer Fragen. Während sich Guttentag durch Veröffentlichungen und Seminare vor allem zu diesen Gebieten qualifizierte, nahm die von Anfang an geringe Zahl seiner Studenten kontinuierlich ab ${ }^{51}$. 1942 bot er der Universität im Rahmen der «national emergency» des Zweiten Weltkriegs an, seinen elektiven Homöopathie-Kurs zu streichen ${ }^{52}$, was sich allerdings aufgrund der auf dem Abkommen mit dem College beruhenden Lehrverpflichtung der Universität aus juristischer Sicht als problematisch erwies.

Als Guttentag 1958 seine Beförderung zum Full Professor beantragte, wurde ihm bzw. dem Hahnemann Medical College of the Pacific von seiten der Universität zur Auflage gemacht, sich mit einer Änderung der Bezeichnung der Professur einverstanden zu erklären - da die Homöopathie inzwischen ein so kleines Gebiet sei, dass sich dafür keine volle Professur rechtfertigen liesse. Aufgrund einer nachträglich undurchsichtigen und später tatsächlich angezweifelten Abstimmung nur einzelner Vertreter des Hahnemann Medical College of the Pacific stimmten diese schliesslich der Umbenennung der Professur in «Samuel Hahnemann Professor of Medical Philosophy» zu, womit das Wort «Homöopathie» fortan eliminiert war ${ }^{53}$. 1962 wurde Guttentag nun Full Professor, ab 1967 bezog er als weiterhin affilierter Emeritus ein reduziertes Gehalt, 1990 wurde er schliesslich ganz 
emeritiert und im Januar 1992 verstarb er. Seine aus Universitätsmitteln mitfinanzierte Stelle wurde inzwischen gestrichen und der noch verbliebene Homeopathy Teaching Fund dem Department of the History of Health Sciences übergeben - zur Verwendung für Gastvorträge zur Geschichte der Homöopathie o. ä.

Im Zusammenhang mit Guttentags Beförderung zum Full Professor, die für die Homöopathen gleichzeitig den Verlust ihres Einflusses an der Universität bedeutete, gründete Elsa K. Engle 1959 die «California Women's Homeopathic Association», deren anfangs ca. 150 Mitglieder in den folgenden Jahrzehnten beträchtliche Geldmittel für homöopathische Forschung beschafften ${ }^{54}$. Vermittelt durch Guttentag wurden der University of California, San Francisco, folgende Stiftungen überreicht: 1974 \$25000 für ein «Hahnemann Lectureship in Correlative Pharmacology» ${ }^{55}$ (dessen Erträge für bisher sieben Gastvorträge, 1975-1989, verwendet wurden), $1980 \$ 30000$ für «Research on the Delineation of Hahnemann's Simile Phenomenon» ${ }^{56}$, $1983 \$ 17000$ für ein «Scholarship for Student Research in the Field of Homeopathy» ${ }^{57}$ sowie 1990 weitere $\$ 17500$ für «Scholarly Studies in the Field of Clinical Homeopathy» ${ }^{58}$. Diese nach wie vor in der UCSF vorhandenen Mittel blieben bisher weitgehend unangetastet - angeblich mangels geeigneter und qualifizierter Anträge für konkrete Forschungsvorhaben.

Nachdem das bedeutungslos gewordene Hahnemann Medical College of the Pacific in den 70er Jahren von seinem verbliebenen Vorstand nicht mehr weiterbetrieben worden und damit juristisch nicht mehr existent war, versuchte 1982 der in den 50er Jahren eingewanderte deutsche homöopathische Arzt Frederic W.Schmid, dieses im Alleingang zu revitalisieren. Durch die Nachzahlung der Steuern der letzten Jahre (ca. \$50) schaltete er auf legale Weise den früheren Vorstand (zu dem auch Guttentag gehörte) aus und machte sich selbst so zum Präsidenten des College. In dieser Funktion versuchte er lange vergeblich, an der Universität wieder einen HomöopathieKurs für Studenten aufzubauen, bis ihm dies 1983 - trotz der Universität gegenüber geäusserter Bedenken von seiten Guttentags - schliesslich erlaubt wurde. Nach einigen Monaten Lehrtätigkeit starb Frederic Schmid allerdings 1984 - am Abend nach seiner letzten Vorlesung - an Herzversagen ${ }^{59}$.

Die jüngste Geschichte der Homöopathie in San Francisco beginnt etwa Mitte der 70er Jahre, als vor dem Hintergrund eines sich breit machenden «New-Age»-Bewusstseins vor allem in Berkeley ein stark vermehrtes Interesse an alternativen Heilmethoden entstand. So wurde von den «neuen» homöopathischen Ärzten der Bay Area etwa Georgos Vithoulkas aus Athen, 
der 1966 am Homeopathic Medical College in Bombay/Indien graduiert worden war, 1978 Gastvorlesungen an der UCSF sowie an der California Academy of Sciences vermittelt, die in der Presse einiges Aufsehen erregten ${ }^{60}$. 1977 war bereits die homöopathische Hering Clinic in Berkeley gegründet worden, aus der 1985 die grössere Hahnemann Medical Clinic, ebenfalls in Berkeley, hervorging, die sich 1992 erneut vergrösserte und nach Albany, CA, umzog ${ }^{61}$. An der UCSF unterrichtet derzeit Michael Carlston, Assistant Clinical Professor am Department of Family and Community Medicine der Universität ein «Family Practice Residency Program» im Sonoma County Hospital in Santa Rosa, CA, in dem er interessierte Residents (vergleichbar mit Ärzten in der Weiterbildung zum Allgemeinarzt) in die Grundlagen der homöopathischen Anamnese und Therapie einführt. Im März 1992 schliesslich fand in San Francisco die Jahreskonferenz des «National Center for Homeopathy» statt, an der etwa 450 Personen teilnahmen, von denen zwar über die Hälfte medizinische Laien waren, die 72 anwesenden Ärzte für ihre Teilnahme aber dennoch C.ME-Credits angerechnet bekamen ${ }^{62}$.

\section{Zusammenfassung und Ausblick}

Die bisher erfassten Aspekte der Geschichte der Homöopathie weisen darauf hin, dass es weniger wissenschaftsimmanente Gründe (etwa Studien zum Wirksamkeitsnachweis der Homöopathie o. ä.) als vielmehr eine Reihe von historischen, soziologischen, politischen, kulturellen und ökonomischen Bedingungen waren, die die Entwicklung (Aufstieg, Fall und Weiterbestehen) dieser Heilmethode in den USA bestimmten. Neben den Ärzten und ihren Gesellschaften und Institutionen kam dabei auch den Laien (Patienten, Geistlichen, Lehrern usw.) und ihren Organisationen eine nicht unbeachtliche Rolle zu. Berücksichtigt man zudem auch die der homöopathischen Lehre als solcher prinzipiell immanenten Schwierigkeiten und Widersprüche sowie den epistemologischen Unterschied ihres Ansatzes von dem der regulären Pharmakologie, der von Streitern beider Seiten nicht immer klar gesehen wurde, so wird deutlich, dass die Geschichte der Homöopathie sicherlich in Form eines Ineinandergreifens verschiedenster Faktoren zu schreiben sein wird, dem einfache Be- oder Entschuldigungen einzelner Parteien nicht gerecht werden können. Da gerade auch die jüngsten Entwicklungen etwa in der Bay Area zeigen, dass die von vielen bereits tot geglaubte Homöopathie auch heute wieder echte Begeisterung zu erwecken vermag, wird diese 
Geschichte wesentlich aber eine unabgeschlossene bleiben, wie die der «regulären» Medizin auch.

\section{Anmerkungen}

11880 etwa gab es in den USA 76 reguläre und 14 homöopathische Medical Colleges [5:1]. An ersteren waren damals 9776 Studenten eingeschrieben, wovon 2673 in diesem Jahr graduierten, an letzteren waren es 1220 Studenten [8:1], von denen 380 graduierten [7:1]. Journal of the American Medical Association [= JAMA] 61 (1913), S. $599 \mathrm{f}$. Vergleichende Jahresstatistiken finden sich in JAMA 49 (1907) bis $J A M A 93$ (1929).

2 Martin Kaufman: Homeopathy in America. The Rise and Fall of a Medical Heresy. Baltimore \& London 1971, S. 174-185.

3 Ifeoma Ikenze: Homeopathy Branches Out. San Francisco Focus 25 (March 1992), S. 32 f. Vgl. dazu Josef M.Schmidt: Bericht über die Jahres-Konferenz des National Center for Homeopathy im März 1992 in San Francisco. Allgemeine homöopathische Zeitung 238 (1993), S. 34-38.

4. Vgl. hierzu Josef M. Schmidt: Die philosophischen Vorstellungen Samuel Hahnemanns bei der Begründung der Homöopathie (bis zum Organon der rationellen Heilkunde, 1810). München 1990.

5 Thomas Lindsley Bradford: Homoeopathy in New York/in Pennsylvania. In: History of Homoeopathy and its Institutions in America. Ed. by William Harvey King. New York \& Chicago 1905, Bd.1, S.44-75/111-165. Pemberton Dudley: Hahnemann Medical College and Hospital of Philadelphia. In: History of Homoeopathy..., Bd. 2, S. $37 \mathrm{ff}$.

6 Harris L. Coulter: Divided Legacy. Science and Ethics in American Medicine 1800-1910. Berkeley, California, 1982, S. 5 ff.

7 Lester S. King (Ed.): American Medicine Comes of Age 1840-1920. American Medical Association 1984, S.9-21. Stanley Joel Reiser, Arthur J. Dyck, William J. Curran (Ed.): Ethics in Medicine. Historical Perspectives and Contemporary Concerns. Cambridge, Massachusetts \& London, England 1977, S. 26-34.

8 William G. Rothstein: American Physicians in the Nineteenth Century. From Sects to Science. Baltimore \& London 1972, S.236.

9 Vgl. dazu Francis R. Packard: History of Medicine in the United States. New York \& London 1963, S. 1216-1218. Frederick C. Waite: American Sectarian Medical Colleges before the Civil War. Bulletin of the History of Medicine 19 (1946), S. 163.

10 Rothstein: American Physicians (s. Anm.8), S.159-161. Robert C. Fuller: Alternative Medicine and American Religious Life. New York \& Oxford 1989, S. 22-26. John S. Haller: American Medicine in Transition 1840-1910. Urbana, Chicago \& London 1981, S. 118f. u.a.

11 Anthony Campbell: The Two Faces of Homoeopathy. London 1984, S.90-95. Elinore Peeble: Homeopathy and the New Church. In: Emanuel Swedenborg. A Continuing Vision. Ed. by Robin Larsen. New York 1988, S.468-472.

12 Vgl. etwa Joseph F. Kett: The Formation of the American Medical Profession. New Haven \& London 1968, S. 140.

13 Harris L. Coulter: Homoeopathic Influences in Nineteenth-Century Allopathic Therapeutics. Washington, D. C. 1973. 
14. Martin Kaufman: Homeopathy in America. The Rise and Fall and Persistence of a Medical Heresy. In: Other Healers. Unorthodox Medicine in America. Ed. by Norman Gevitz. Baltimore \& London 1988, S. 105-109. Coulter: Divided Legacy (s. Anm. 6), S. 241-276.

15 Phillip A. Nicholls: Homoeopathy and the Medical Profession. London, New York, Sydney 1988, S. 194-197.

16 Rosemary Stevens: American Medicine and the Public Interest. New Haven \& London 1971, S. 55-58.

17 Vgl. dazu W. Bruce Fye: The Origin of the Full-time Faculty System. JAMA 265 (1991), S. 1555-1562.

18 Vgl. dazu die programmatische Rede des damaligen Präsidenten der AMA, Nathan Smith Davis: Address on the Present Status and Future Tendencies of the Medical Profession in the United States. JAMA 1 (1883), S. 33-42.

19 Stevens: American Medicine (s. Anm. 16), S. 59.

20 Coulter: Divided Legacy (s. Anm. 6), S.417-419.

21 Abraham Flexner: Medical Education in the United States and Canada. A Report to the Carnegie Foundation for the Advancement of Teaching. (Carnegie Foundation Bull. No.4) New York 1910.

22 Stevens: American Medicine (s. Anm. 16), S. 68-73.

23 Coulter: Divided Legacy (s. Anm. 6), S. 449f. u. $463 \mathrm{f}$.

24 Ebd., S. 447. Flexner-Report (s. Anm. 21), S. 156-166: The Medical Sects.

25 Vgl. dazu die in Anm. 1 erwähnten Jahresstatistiken des JAMA.

26 Martin R.Lipp: Medical Landmarks USA. New York u. a. 1991, S. 157-159: Hahnemann University Medical Center.

27 Kaufman: Homeopathy in America (s. Anm. 14), S.113.

28 Vgl. Schmidt: Jahreskonferenz des NCH (s. Anm. 3).

29 James Hazelwood: Early M. D.s Served Their Patients Well. Oakland Tribune [California], June 16, 1974, S.4. u. 6.

30 J.M. Selfridge: An Epitome of the Early History of Homoeopathy in California. Reprint from the Pacific Coast Journal of Homoeopathy 9 (1901), S. 181-195. Henry Harris: California's Medical Story. San Francisco 1932, S.197-199.

31 James William Ward: Hahnemann Medical College of the Pacific. In: King (Ed.): History of Homoeopathy (s. Anm. 5), Bd. 3, S. 214-239 [hier: 214-221] sowie Pacific Coast Journal of Homeopathy 26 (1915), S. 305-311, 390-400 u. 455-460.

32 Ebd., S.222-226.

33 Ebd., S.232f.

34 A History of Marshal Hale Memorial Hospital (Formerly Hahnemann Hospital of San Francisco) [Masch.schr. Manuskript]. San Francisco 1987, S.2. Marshal Hale Memorial Hospital. Commemorated March 25, 1975 [Prospekt], S.3. University of California, San Francisco, Library, Special Collections [= UCSF-SC].

35 Flexner-Report (s. Anm.21), S.194f.

36 H. College of the Pacific, H. Hospital [Manuskript], S. 1-2. UCSF-SC. Minutes. Meeting of the Board of Directors of Hahnemann Medical College of the Pacific, Sept. 28, 1916, S.5254. UCSF-SC, MSS 91-5.

37 Brief von Herbert C. Moffit [1913-1919 Dean, Univ. of Calif., Medical School] an Benjamin Ide Wheeler [1899-1919 President, Univ. of Calif.], Sept. 6, 1915. University of California, 
Berkeley, Archives, President's Files $[=U C B-P F]$. Vgl. auch William Boericke: Inaugural Lecture, Department of Homeopathy. Pacific Coast Journal of Homeopathy 27 (1916), S. 172-185.

38 Brief von James W. Ward [Dean, Hahnemann Medical College of the Pacific] an Benjamin Ide Wheeler, April 18, 1917, UCB-PF.

39 Memorandum for the Finance Committee, Jan. 6, 1921, UCB-PF.

40 Pacific Coast Journal of Homoeopathy 33 (1922), S. 29 u. 65 sowie 34 (1923), S. 5.

41 Minutes of the Meeting of the Board of Directors of the Hahnemann Hospital, Sept. 5, 1918 \& Jan. 9, 1919, S. 171-181. UCSF-SC, MSS 91-5.

42 Vgl. History of MHMH sowie MHMH (s. Anm. 34).

43 Brief von H.H. Benedict an Monroe E. Deutsch [Provost, Univ. of Calif.], Nov. 16, 1933 sowie die Briefe von William J. Kerr [Prof. of Medicine, Univ. of Calif., Medical School] to Langley Porter [1927-1937 u. 1939-1940 Dean, Univ. of Calif., Medical School], Dec. 14, 1933 \& July 6, 1934. UCB-PF.

44 Brief von Thomas McGavack an William J. Kerr, July 2, 1934, UCB-PF.

45 Thomas Hodge McGavack: An Open Letter to the Homoeopathic Profession. Pacific Coast Journal of Homoeopathy 45 (1934), S.424-440.

46 Brief von Robert G. Sproul [1929-1958 President, Univ. of Calif.] an John L. MeNab [Attorney], Jan. 17, 1939. UCB-PF.

47 So war etwa William Wallace Campbell [1923-1929 Präsident der University of California] ein früherer Patient von William Boericke. Pacific Coast Journal of Homoeopathy 34 (1923), S. 6.

48 So heisst es etwa im Memorandum to the President, Oct. 31, 1935, UCB-PF: «We determined that to all of us the proposals of Mr. Jordan were distinctly unacceptable»...

49 Memorandum to the President, Nov. 30, 1935, UCB-PF. New Contract between the University of California Medical School and the Hahnemann Medical College of the Pacific. Journal of the American Institute of Homeopathy 29 (1936), S. 769-773.

50 Vgl. dazu Otto E. Guttentag: Four Years of Homeopathy at the University of California Medical School. The Hahnemannian Monthly 76 (1941), S. 155-160.

51 Interviews mit Otto E. Guttentag (1900-1992) am 3., 6. und 10.10.1989 in San Francisco sowie am 2.12.1991 in Oakland.

52 Brief von Otto E. Guttentag an Francis S. Smyth [1942-1954 Dean, Univ. of Calif., Medical School], March 16, 1942. UCB-PF.

53 Im Protokoll der diesbezüglichen Sitzung, in der anscheinend nur vier Personen anwesend waren, heisst es lapidar: «After a short discussion, it was moved, seconded and carried unanimously that the contract offered by the regents of the University of California be accepted as offered and duly signed by the president and the secretary. This ended this historical event striking out the name of Homeopathy from our contract with the University of California». Minutes of the Meeting of the Members of the Hahnemann Medical College of the Pacific, Febr.6, 1958. Kopie aus dem Nachlass von Frederic W. Schmid, San Francisco.

54. Interviews mit Elsa K. Engle (1895-1994) am 7.10.1989, 11.12.1991, 17.2. u. 6.6.1992 in San Francisco.

55 Minutes. Meeting of California Women's Homeopathic Association [= CWHA], Dec. 10, 1974, S. 111. UCSF-SC. 
56 Minutes. Meeting of CWHA, Dec. 3, 1980, S.135-138. Vgl. dazu Minutes. Board of Directors Meeting [CWHA], April 8, 1981, S.45f. UCSF-SC.

57 Minutes. Meeting of CWHA, Dec. 12, 1983, S. 145 f. UCSF-SC.

58 Brief von Elsa K. Engle an Jere E. Goyan [Dean, UCSF, School of Pharmacy], March 26, 1990 sowie Dankschreiben von Jere E. Goyan an Elsa K. Engle, Aug. 1, 1990, UCSF-SC.

59 Interviews mit der Witwe Irmgard Schmid-Maybach am 19.12.1991, 3.2. u. 8.3.1992 in San Francisco.

60 Vgl. dazu Richard Grossinger: Planet Medicine. From Stone Age Shamanism to Postindustrial Healing. Boulder \& London 1982, S. 241-244.

61 Interview mit Roger Morrison, ltd. Arzt der Klinik, am 7.7.1992 in Albany, California.

62 Vgl. Schmidt: Jahreskonferenz des NCH (s. Anm. 3).

Dr. med. Dr. phil. Josef M. Schmidt

Institut für Geschichte der Medizin

der Ludwig-Maximilians-Universität München

Lessingstrasse 2

D-80336 München 